\title{
Serviço de saúde procurado pelas pessoas com sintomas da tuberculose
}

Health service searched by people with tuberculosis symptoms

Servicio de salud utilizado por las personas con síntomas de la tuberculosis

\author{
Martina Dias da Rosa Martins', Natali Basílio Valerão ${ }^{I I}$, Jéssica Oliveira Tomberg ${ }^{I I}$ \\ Lílian Moura de Lima Spagnolo ${ }^{\mathrm{V}}$, Luciana Nunes Soares ${ }^{\mathrm{v}}$, Roxana Isabel Cardozo \\ Gonzales $^{\mathrm{VI}}$
}

Resumo: Objetivo: identificar o perfil das pessoas com tuberculose em relação ao comportamento de busca por atendimento diante dos primeiros sintomas da doença. Método: estudo quantitativo desenvolvido em quatro municípios do Rio Grande do Sul entre 2013 e 2014, com aplicação de questionário estruturado às pessoas com tuberculose pulmonar que iniciaram o tratamento no período. Análise dos dados realizada no software Statistica 12, utilizando-se estatística descritiva. Resultados: o serviço de saúde mais procurado foi o pronto atendimento (35,7\%). Quanto ao perfil, houve predomínio do sexo masculino (58,7\%), cor da pele branca $(59,8 \%)$, viviam sem companheiro $(67,4 \%)$, escolaridade inferior a 8 anos $(75,0 \%)$, emprego formal $(41,3 \%)$, renda de 1 a 2 salários mínimos (43,5\%). Conclusão: houve diferenças no perfil de acordo com o comportamento de busca por atendimento, e destaca-se que conhecê-lo é essencial para o planejamento de ações de diagnóstico precoce.

Descritores: Tuberculose; Acesso aos Serviços de Saúde; Pesquisa sobre Serviços de Saúde; Atenção Primária à Saúde

\footnotetext{
I Graduanda. Enfermagem na Universidade Federal de Pelotas. Pelotas, RS, Brasil. E-mail: martinadrm@hotmail.com ORCID: https://orcid.org/0000-0002-8835-6284

II Enfermeira. Residente Hospital de Clínicas. Porto Alegre, RS, Brasil. E-mail: natalibasilio@hotmail.com ORCID: https:/orcid.org/0000-0002$2920-837 \mathrm{X}$

III Doutora em Ciências. Universidade Federal de Pelotas. Pelotas, RS, Brasil. E-mail: jessicatomberg@hotmail.com ORCID: https://orcid.org/0000-0002-1194-9970

IV Docente na Faculdade de Enfermagem da Universidade Federal de Pelotas. Doutora em Ciências. Pelotas, RS, Brasil. E-mail: lima.lilian@gmail.com ORCID: https://orcid.org/0000-0003-2070-6177

Enfermeira da Secretaria Municipal de Saúde de Pelotas. Mestre em Ciências da Saúde. Pelotas, RS, Brasil. E-mail: Luciana.nunes.soares@gmail.com ORCID: https://orcid.org/0000-0003-2645-0344

VI Professor adjunto na Faculdade de Enfermagem da Universidade Federal de Pelotas. Pós-doutora em Enfermagem de Saúde Pública pela Escola de Enfermagem de Ribeirão Preto, SP. Pelotas, RS, Brasil. E-mail: Roxana_cardozo@hotmail.com ORCID: https://orcid.org/0000-00017180-897X
} 
Serviço de saúde procurado pelas pessoas com sintomas da tuberculose $\mid 2$

\begin{abstract}
Aim: to identify the profile of people with tuberculosis in relation to the behavior of search for care against the first symptoms of the disease. Method: quantitative study developed in four cities of Rio Grande do Sul, between 2013 and 2014, with the application of a structured questionnaire to people with pulmonary tuberculosis who began treatment within the period. Data analysis was performed in Statistica 12 software, using descriptive statistics. Results: the most sought after health service was prompt care (35.7\%). Regarding the profile, there was a predominance of males (58.7\%), white skin color (59.8\%), who lived without a partner (67.4\%), education less than 8 years $(75.0 \%)$, formal employment (41.3\%), income from 1 to 2 minimum wages (43.5\%). Conclusion: there were differences in the profile according to the behavior of search for care, and it is noted that knowing it is essential for the planning of early diagnosis actions.
\end{abstract}

Descriptors: Tuberculosis; Access to Health Services; Research on Health Services; Primary Health Care

Resumen: Objetivo: identificar el perfil de las personas con tuberculosis con relación al comportamiento de búsqueda por atención a partir de los primeros síntomas de la enfermedad. Método: estudio cuantitativo, desarrollado en cuatro municipios del Rio Grande do Sul, entre 2013 y 2014, por medio de la aplicación de cuestionario estructurado a las personas con tuberculosis pulmonar, que iniciaron el tratamiento en el período. Análisis de los datos realizada por el software Statistica 12, utilizando estadística descriptiva. Resultados: el servicio de salud más buscado fue el de pronta atención (35,7\%). Cuanto al perfil hubo predominio del sexo masculino $(58,7 \%)$, color de piel blanca $(59,8 \%)$, vivían sin compañero $(67,4 \%)$, escolaridad inferior a 8 años $(75,0 \%)$, empleo formal (41,3\%), renta de 1 a 2 salarios mínimos (43,5\%). Conclusión: hubo diferencias en el perfil de acuerdo con el comportamiento de búsqueda por atención, y se destaca que conocerlo es esencial para la planificación de acciones de diagnóstico precoz.

Descriptores: Tuberculosis; Acceso a los servicios de salud; Investigación sobre servicios de salud; Atención primaria a la salud

\title{
Introdução
}

A tuberculose é uma doença infectocontagiosa, considerada pela Organização Mundial de Saúde como emergência de saúde pública. Anualmente são notificados cerca de 10 milhões de casos novos em todo o mundo e mais de milhão de pessoas vão ao óbito por causa da doença. ${ }^{1}$ O Brasil está dentre os países que apresentam altas taxas de concentração da tuberculose. Em 2015, foram registrados 63.189 casos novos da doença, representando uma taxa de incidência de 30,9/100 mil hab., o número de óbitos registrados foi de 4.543, apresentando uma taxa de mortalidade de 2,2/100 mil hab. ${ }^{2-3}$

As estratégias para o controle da tuberculose centram-se, prioritariamente, na interrupção da cadeia de transmissão, e para isso é essencial que ocorra a detecção precoce dos casos, com a busca ativa e passiva dos sintomáticos respiratórios (pessoas com tosse por mais de 
3 | Martins MDR, Valerão NB, Tomberg JO, Spagnolo LML, Soares LN, Gonzales RIC

três semanas), o diagnóstico precoce e o tratamento em tempo oportuno. ${ }^{4}$ Assim, o primeiro serviço de saúde procurado pelas pessoas com sintomas da tuberculose necessita demonstrar agilidade no processo de detecção dos casos. ${ }^{5}$

Nesse contexto, as unidades de Atenção Primária a Saúde são os pontos de atenção considerados prioritários para a detecção, pois a tuberculose é uma condição sensível a este nível, ou seja, têm a capacidade de resolutividade do problema, encaminhando para as demais unidades somente quando necessário. Ademais, pelo seu processo de trabalho e proximidade com a comunidade, torna-se preparada para garantir a continuidade da atenção. ${ }^{4,6-7}$

Contudo, estudos realizados revelam que as unidades de saúde escolhidas pelos usuários para o primeiro atendimento não é a Atenção Primária a Saúde, mas sim as unidades de Pronto Atendimento. ${ }^{6-7}$ Situação essa que compromete o funcionamento da rede de serviços de saúde, além de dificultar a efetividade de uma assistência continua e integral.

A escolha por uma unidade de saúde para atendimento relaciona-se com experiências prévias, facilidade de acesso, sinais e sintomas apresentados, garantia de atendimento e resolutividade do problema. Além disso, a cultura do modelo curativista ainda influência nesse processo..$^{7-8}$

Identificar o primeiro serviço procurado pelas pessoas com sintomas da tuberculose e realizar o mapeamento das características sociodemográficas relevante, uma vez que permite direcionar a busca e identificar a tuberculose na demanda de forma ágil e precoce. Este aspecto é de grande valor do ponto de vista epidemiológico e social da doença. A fim de responder as questões de pesquisa, foram elaboradas as questões qual o perfil das pessoas com tuberculose? qual seu comportamento de busca por atendimento nos primeiros sintomas? Assim, o presente artigo tem como objetivo identificar o perfil das pessoas com tuberculose em relação ao comportamento de busca por atendimento diante dos primeiros sintomas da doença. 
Serviço de saúde procurado pelas pessoas com sintomas da tuberculose $\mid 4$

\section{Método}

Trata-se de um estudo quantitativo, de corte transversal, desenvolvido em quatro municípios prioritários para o controle da tuberculose no estado Rio Grande do Sul (Pelotas, Sapucaia do Sul, Canoas e Santa Cruz do Sul).

O Estado do Rio Grande do Sul em 2015 apresentou uma taxa de incidência de 41/100 mil habitantes, sendo superior a taxa nacional de 33,2/100 mil hab. O município de Canoas contribuiu para esses indicadores com a notificação de 269 casos, Pelotas com 287, Santa Cruz do Sul com 57 e Sapucaia do Sul com 146. Estes municípios estão dentre os quinze prioritários para as ações de controle da tuberculose. ${ }^{9}$

A coleta de dados foi prospectiva e ocorreu nos ambulatórios do Programa Municipal de Controle da Tuberculose dos municípios estudados. A amostra foi intencional, uma vez que foi considerada a totalidade das pessoas que iniciavam o tratamento para tuberculose no período de agosto de 2013 a julho de 2014. Os critérios de exclusão foram: pessoas menores de 18 anos de idade; que não podiam comunicar-se verbalmente ou com déficit cognitivo; e as que estavam institucionalizadas. Aqueles que atendiam os critérios para participação no estudo eram convidadas por entrevistadores a responder o formulário estruturado de entrevista, em sala privativa.

O formulário estruturado possuía perguntas direcionadas ao uso dos serviços de saúde e ações realizadas por esses. O referido instrumento de coleta de dados passou por avaliação de pesquisadores na área temática da tuberculose de três universidades do Sul do Brasil, posteriormente foi realizado o teste piloto em um município prioritário para tuberculose do estado de Santa Catarina.

As variáveis referentes a características dos entrevistados foram sexo, média de idade, renda familiar (menor que 1 salário mínimo, entre 1 até 2 , maior que 2 até 3 , maior que 3 até 4 e mais de 4 salários mínimo), cor da pele (branca e não branca), se possuía companheiro, 
5 | Martins MDR, Valerão NB, Tomberg JO, Spagnolo LML, Soares LN, Gonzales RIC

escolaridade (menor ou igual a oito anos de estudo ou maior que oito anos de estudo), fonte de renda (emprego formal, desempregado, trabalho autônomo, aposentado, auxílio doença). Ademais, foram analisadas variáveis referentes as características comportamentais e comorbidades, sendo outras doenças (sim e não), uso de álcool (nunca, quase nunca, às vezes, quase sempre e sempre), fumo (nunca, quase nunca, às vezes, quase sempre e sempre) e drogas (sim e não).

Para caracterizar o primeiro serviço de saúde procurado, a variável tipo de serviço foi estratificada e agrupada em Atenção Primária a Saúde, incluindo as Unidades Básica de Saúde, o Programa de Agentes Comunitários de Saúde e Estratégia de Saúde de Família; Serviços privados incluindo os hospitais privados e consultórios particulares; o ambulatório de referência para atenção à tuberculose, pronto atendimento, e outros serviços incluindo os hospitais públicos e clinicas especializadas.

Para as análises dos dados foram desconsiderados os questionários dos participantes que não haviam respondido o primeiro tipo de serviço que procuraram. Assim, dos 343, finalizou-se com 258 respondentes. Para a análise das variáveis em escala likert (nunca, quase nunca, às vezes, quase sempre e sempre), em valores de um a cinco, foi estipulado uma classificação em que o valor 1 (nunca) foi considerado a resposta negativa e os demais valores (2, 3 e 4 ) como resposta positiva.

As análises foram feitas no programa Statistica12 da Stat Soft, realizando cruzamento das características das pessoas com tuberculose e o tipo de serviço procurado, apresentando as frequências relativas e absolutas e o teste qui-quadrado, considerando significância estatística valores de $p<0,05$.

$\mathrm{Na}$ realização deste estudo, foram respeitados os preceitos éticos da Resolução 466/12 do Conselho Nacional de Saúde. ${ }^{10}$ Todos os entrevistados concordaram em participar, assinaram o Termo de Consentimento Livre e Esclarecido em duas vias. A coleta de dados só foi iniciada 
Serviço de saúde procurado pelas pessoas com sintomas da tuberculose $\mid 6$

após a submissão e aprovação do projeto pelo Comitê de Ética em Pesquisa, com parecer favorável número 310.801 em 15 de julho de 2013.

\section{Resultados}

Do total dos 258 entrevistados, 40,3\% (104) eram do município de Canoas, 34,5\% (89) de Pelotas, 15,5\% (40) de Santa Cruz do Sul e 9,7\% (25) de Sapucaia do Sul. Com relação as características gerais da população em estudo, predominou o sexo masculino com 62,8\% (162), a cor da pele branca com 60,9\% (157), os que vivem sem companheiro com $62,4 \%$ (161), os que estudaram menos de oito anos com 75,2\% (194), e aqueles com renda de 1 a 2 salários mínimos com 38,8\% (100). A média de idade foi de 44,4 anos (DP=16,3) e variou de 19 a 87 anos.

O primeiro serviço de saúde procurado foi o Pronto Atendimento (PA) com 35,7\% (92) dos entrevistados, seguido por 25,2\% (65) que procuram a Atenção Primária à Saúde (APS), 15,9\% (41) os serviços particulares, 12,4\% (32) buscaram outros serviços e 10,9\% (28) procuraram diretamente ao ambulatório de referência para a tuberculose dos municípios em estudo.

$\mathrm{Na}$ Tabela 1, ao verificar os indivíduos que buscaram pela APS e o ambulatório de referência como primeiro serviço no processo de diagnóstico da tuberculose observa-se, respectivamente, o predomínio de homens $(64,6 \%$ e $75 \%)$, em relação à cor da pele verifica-se distribuição paritária entre branca e não branca $(49,2 \%$ e 50\%). Houve concentração de indivíduos que vivem sem companheiro(a) (73,9\% e 71,4\%), com escolaridade inferior a oito anos (84,6\% e 82,1\%), e renda familiar inferior a dois salários mínimos (66,2\% e 64,3\%). Quanto à fonte de renda, houve $40 \%$ de desempregados que buscaram a APS, e distribuição igualitária entre emprego formal e desempregados dentre os que buscaram o Ambulatório de referência, com $35,7 \%$.

Tabela 1 - Características sociodemográficas das pessoas com tuberculose de acordo com o primeiro serviço de saúde procurado, Rio Grande do Sul, Brasil, 2014 (N=258). 
7 | Martins MDR, Valerão NB, Tomberg JO, Spagnolo LML, Soares LN, Gonzales RIC

\begin{tabular}{|c|c|c|c|c|c|c|c|c|c|c|c|}
\hline \multirow[t]{2}{*}{ Características } & \multicolumn{2}{|c|}{ APS (65) } & \multicolumn{2}{|c|}{$\begin{array}{c}\text { Amb. Ref. } \\
(28)\end{array}$} & \multicolumn{2}{|c|}{ PA (92) } & \multicolumn{2}{|c|}{$\begin{array}{l}\text { S.Privado } \\
(41)\end{array}$} & \multicolumn{2}{|c|}{ Outros (32) } & \multirow[t]{2}{*}{$P$} \\
\hline & $\mathrm{n}$ & $\%$ & $\mathrm{~N}$ & $\%$ & $\mathrm{~N}$ & $\%$ & $\mathrm{~N}$ & $\%$ & $\mathrm{~N}$ & $\%$ & \\
\hline \multicolumn{12}{|l|}{ Sexo } \\
\hline Feminino & 23 & 35,4 & 7 & 25,0 & 38 & 41,3 & 18 & 43,9 & 10 & 31,2 & \multirow{2}{*}{0,432} \\
\hline Masculino & 42 & 64,6 & 21 & 75,0 & 54 & 58,7 & 23 & 56,1 & 22 & 68,7 & \\
\hline \multicolumn{12}{|l|}{ Cor da pele } \\
\hline Branca & 33 & 50,8 & 14 & 50,0 & 55 & 59,8 & 32 & 78,0 & 23 & 71,9 & \multirow{2}{*}{0,027} \\
\hline Não Branca & 32 & 49,2 & 14 & 50,0 & 37 & 40,2 & 9 & 22,0 & 9 & 28,1 & \\
\hline \multicolumn{12}{|l|}{$\begin{array}{l}\text { Vive com } \\
\text { companheiro(a) }\end{array}$} \\
\hline Não & 48 & 73,9 & 20 & 71,4 & 62 & 67,4 & 15 & 36,6 & 16 & 50,0 & \multirow{2}{*}{0,001} \\
\hline $\operatorname{Sim}$ & 17 & 26,1 & 8 & 28,6 & 30 & 32,6 & 26 & 63,4 & 16 & 50,0 & \\
\hline \multicolumn{12}{|l|}{ Escolaridade } \\
\hline$\leq 8$ anos & 55 & 84,6 & 23 & 82,1 & 69 & 75,0 & 20 & 48,8 & 27 & 84,4 & \multirow{2}{*}{0,000} \\
\hline$>8$ anos & 10 & 15,4 & 5 & 17,9 & 23 & 25,0 & 21 & 51,2 & 5 & 15,6 & \\
\hline \multicolumn{12}{|l|}{ Fonte de renda } \\
\hline Emprego formal & 17 & 26,2 & 10 & 35,7 & 38 & 41,3 & 24 & 58,5 & 11 & 34,4 & \multirow{5}{*}{0,085} \\
\hline Desempregado & 26 & 40,0 & 10 & 35,7 & 27 & 29,3 & 5 & 12,2 & 14 & 43,7 & \\
\hline $\begin{array}{l}\text { Trabalho } \\
\text { autônomo }\end{array}$ & 14 & 21,5 & 4 & 14,3 & 15 & 16,3 & 2 & 4,9 & 3 & 9,4 & \\
\hline Aposentadoria & 6 & 9,2 & 3 & 10,7 & 10 & 10,9 & 8 & 19,5 & 3 & 9,4 & \\
\hline Auxílio doença & 2 & 3,1 & 1 & 3,6 & 2 & 4,9 & 2 & 4,9 & 1 & 3,1 & \\
\hline \multicolumn{12}{|l|}{$\begin{array}{l}\text { Renda familiar } \\
\text { (em salários } \\
\text { mínimos)* }\end{array}$} \\
\hline$<1$ & 20 & 30,8 & 5 & 17,9 & 22 & 23,9 & 4 & 9,8 & 7 & 21,9 & \multirow{5}{*}{0,001} \\
\hline 1 a 2 & 23 & 35,4 & 13 & 46,4 & 40 & 43,5 & 8 & 19,5 & 16 & 50,0 & \\
\hline 2 a 3 & 18 & 27,7 & 7 & 25,0 & 17 & 18,5 & 14 & 34,1 & 8 & 25,0 & \\
\hline 3 a 4 & 0 & 0,0 & 3 & 10,7 & 4 & 4,3 & 6 & 14,6 & 1 & 3,1 & \\
\hline 4 ou mais & 4 & 6,1 & 0 & 0,0 & 9 & 9,8 & 9 & 21,9 & 0 & 0,0 & \\
\hline
\end{tabular}

*Valor do salário mínimo em 2014: R\$ 724,00

APS: Atenção Primária à Saúde

Amb. Ref.: Ambulatório de Referência

PA: Pronto Atendimento

S. Privado: Serviços Privados

Dentre os que buscaram pelos serviços de Pronto Atendimento, Serviços Privados e outros serviços (hospitais e ambulatórios públicos), foram verificadas similaridades no perfil, sendo o predomínio de homens $(58,7 \% ; 56,1 \% ; 68,7 \%)$, e de cor da pele branca $(59,8 \% ; 78 \% ; 71,9 \%)$. Quanto a viver com companheiro, verifica-se diferenças e entre os que buscaram o PA $67,4 \%(62)$ 
Serviço de saúde procurado pelas pessoas com sintomas da tuberculose $\mid 8$

não tinham companheiro(a), entre os que buscaram os serviços privados houve predomínio de indivíduos que viviam com companheiro(a) (63,4\%), e entre os outros serviços houve distribuição igualitária.

A escolaridade inferior a oito anos esteve concentrada entre os que procuraram o PA (75\%) e os outros serviços $(84,4 \%)$, já os que buscaram os serviços privados foram em $51,2 \%$ dos casos indivíduos com escolaridade superior a oito anos. Para os que buscaram o PA, houve predomínio de indivíduos com emprego formal (41,3\%), assim como aqueles que buscaram os serviços privados $(58,5 \%)$. A renda familiar inferior a dois salários mínimos foi predominante entre os que buscaram o PA $(67,4 \%)$, e os outros serviços $(71,9 \%)$; já entre os que buscaram serviços privados houve ocorrência de $70,6 \%$ entre os indivíduos com renda superior a dois salários mínimos.

Verificou-se que as variáveis viver com companheiro, escolaridade e renda familiar, apresentaram distribuição estatisticamente significativa em relação ao comportamento de busca pelos serviços de saúde.

Tabela 2 - Características comportamentais e comorbidades das pessoas com tuberculose de acordo com o primeiro serviço de saúde procurado, Rio Grande do Sul, Brasil, 2014 (N=258).

\begin{tabular}{|c|c|c|c|c|c|c|c|c|c|c|c|}
\hline \multirow[t]{2}{*}{ Características } & \multicolumn{2}{|c|}{ APS (65) } & \multicolumn{2}{|c|}{$\begin{array}{c}\text { Amb. Ref. } \\
(28)\end{array}$} & \multicolumn{2}{|c|}{ PA (92) } & \multicolumn{2}{|c|}{$\begin{array}{c}\text { S. Privado } \\
(41)\end{array}$} & \multicolumn{2}{|c|}{$\begin{array}{c}\text { Outros } \\
(32)\end{array}$} & \multirow[t]{2}{*}{$P$} \\
\hline & $\mathrm{N}$ & $\%$ & $\mathrm{~N}$ & $\%$ & $\mathrm{~N}$ & $\%$ & $\mathrm{~N}$ & $\%$ & $\mathrm{~N}$ & $\%$ & \\
\hline \multicolumn{12}{|l|}{ Co-morbidades } \\
\hline $\operatorname{Sim}$ & 26 & 40,0 & 16 & 57,1 & 41 & 44,6 & 21 & 51,2 & 14 & 43,7 & 0,570 \\
\hline Não & 39 & 60,0 & 12 & 42,9 & 51 & 55,4 & 20 & 48,8 & 18 & 56,3 & \\
\hline \multicolumn{12}{|l|}{ Uso de álcool } \\
\hline Não & 50 & 76,9 & 16 & 57,1 & 60 & 65,2 & 27 & 65,9 & 19 & 58,4 & 0,279 \\
\hline $\operatorname{Sim}$ & 15 & 23,1 & 12 & 42,9 & 32 & 34,8 & 14 & 34,1 & 13 & 40,6 & \\
\hline \multicolumn{12}{|l|}{ Uso de tabaco } \\
\hline Não & 39 & 60 & 16 & 57,1 & 50 & 54,3 & 29 & 70,7 & 17 & 53,1 & 0,456 \\
\hline $\operatorname{Sim}$ & 26 & 40 & 12 & 42,9 & 42 & 45,7 & 12 & 29,3 & 15 & 46,9 & \\
\hline \multicolumn{12}{|l|}{ Uso de drogas } \\
\hline Não & 58 & 89,2 & 24 & 85,7 & 83 & 90,2 & 38 & 92,7 & 29 & 90,6 & 0,917 \\
\hline Sim & 7 & 10,8 & 4 & 14,3 & 9 & 9,8 & 3 & 7,3 & 3 & 9,4 & \\
\hline
\end{tabular}

APS: Atenção Primária à Saúde 
Amb. Ref.: Ambulatório de Referência

PA: Pronto Atendimento

S. Privado: Serviços Privados

Na Tabela 2 verifica-se a distribuição das características comportamentais e a existência de comorbidades pelos serviços escolhidos para o primeiro atendimento. A presença de comorbidades ocorreu, prioritariamente, nos grupos que procuraram o primeiro atendimento no ambulatório de referência $(57,1 \%)$ e nos serviços privados $(51,2 \%)$. Quanto ao consumo de substâncias (álcool, tabaco e drogas), observa-se que predominou a negativa dos entrevistados, independendo do serviço procurado. Entretanto, ainda foi referido o consumo de álcool por $33,3 \%$ (86), de tabaco por $41,5 \%$ (107), e de drogas por $10,1 \%$ (26) dos entrevistados.

\section{Discussão}

O primeiro serviço procurado tem papel primordial na detecção da tuberculose e, consequentemente, a interrupção da cadeia de transmissão. Esta procura por atendimento em serviços de saúde é influenciada por diversos fatores, relacionados com aspectos dos usuários e dos serviços de saúde. Facilidades no acesso físico, financeiro, geográfico, estrutural e processual, amparados por um sistema municipal de saúde capaz de proporcionar uma rede de serviços de saúde integrada, têm influência nesse processo. ${ }^{5}$

O PA foi destacado como principal serviço de escolha dos usuários para o atendimento, corroborando com outros estudos realizados no cenário nacional. ${ }^{6-7}$ Fatores associados a essa escolha já foram evidenciados em estudo realizado no Estado de São Paulo, em 2014, o qual entrevistou pessoas com tuberculose. Neste estudo foi destacado o contato anterior com acometidos pela doença, alertaram para a possiblidade dos sintomas apresentados serem de tuberculose, e ainda que as experiências anteriores com o serviço de saúde escolhido foi determinante para o seu comportamento de busca. ${ }^{11}$ 
Serviço de saúde procurado pelas pessoas com sintomas da tuberculose | 10

O comportamento de busca pelo PA em detrimento da APS pode ser justificado por aspectos relacionados às questões culturais, como a espera do agravamento dos sintomas para buscar o primeiro atendimento em saúde, e o predomínio de homens na amostra estudada os quais, geralmente, apresentam resistência em buscar atendimento em saúde nas unidades de APS, conforme verificado em estudo realizado em São José do Rio Preto, São Paulo. ${ }^{6,12}$ Identificou-se a preferência pelos serviços de urgência e emergência devido ao imediatismo e resolutividade em exames, associada as experiências com falhas da rede primária de saúde, tais como a falta de profissionais e de ações de acolhimento, as quais colaboram para tal situação. ${ }^{13-14}$

Quanto a cor da pele, a maioria da população (60,9\%) em estudo se autodeclarou branca, apresentando diferença estatisticamente significante com relação ao tipo de serviço procurado. Entretanto, estudo realizado no Piauí, evidenciou que a maioria das pessoas com tuberculose (70\%) eram pardas. ${ }^{15}$ Cabe ressaltar que tais resultados podem ter relação com a predominância da cor da pele na população do estado/região estudada. No Rio Grande do Sul, 83,22\% da população é autodeclarada de cor branca. ${ }^{16}$

A associação estatística entre o tipo de serviço de saúde procurado e a variável viver com companheiro, ratifica o importante papel da família para o fortalecimento das ações de controle da tuberculose.${ }^{17} \mathrm{O}$ apoio familiar representa um importante suporte social para a pessoa doente de TB durante a busca por atendimento em saúde. Além disso, com a supervisão e estímulo dos familiares, é possível que a pessoa com TB perceba mais brevemente as alterações de saúde promovidas pela doença, favorecendo o diagnóstico precoce. ${ }^{18}$

Quanto ao grau de escolaridade, verificou-se que houve predomínio de entrevistados com menos de 8 anos de estudo, os quais buscaram os serviços públicos de saúde, sendo a APS, o PA e o ambulatório de referência. Esta característica vai ao encontro do observado em estudo de base populacional realizado em Natal, Rio Grande do Norte, entre os anos de 2009 e 2012, assim como em estudo realizado no Paraná. ${ }^{18-19}$ Em ambos, houve predomínio de indivíduos com 
ensino fundamental incompleto entre os acometidos pela TB. Destaca-se a relação entre o grau de escolaridade e a percepção da presença de alterações no estado de saúde, assim como para o entendimento sobre a doença e suas consequências, o que vem a determinar o comportamento de busca pelos serviços de saúde e a adesão ao tratamento. ${ }^{19-20}$ Diante disso, é primordial que os serviços de saúde levem em consideração a influencia destes fatores no enfrentamento da doença, capacitando suas equipes para acolher os usuários de forma que os empodere sobre o autocuidado. ${ }^{21}$

Com relação a fonte de renda, observou-se que as pessoas que buscaram APS e outros serviços constituíram-se, predominantemente, por desempregados, enquanto as que optaram pelo PA e os serviços privados foram as pessoas com emprego formal. Tais resultados podem ser reflexo de que as pessoas com emprego formal, normalmente, optam por atendimento de saúde em período oposto ao horário de trabalho, em virtude do receio de perder o emprego diante faltas e atrasos. ${ }^{19}$ Além disso, as pessoas desempregadas buscam por serviços públicos próximos ao domicílio, visto que apresentam dificuldades em percorrer longas distâncias pelos gastos em deslocamentos com transporte motorizado. ${ }^{22} \mathrm{Em}$ contrapartida, àqueles com maior renda buscaram os serviços privados, resultado já esperado devido ao acesso facilitado às pessoas com maior renda ao serviços privados e planos de saúde. Destaca-se que a opção por serviços pagos envolve uma relação de confiança e aceitabilidade, uma vez que o usuário pode optar pelo serviço ou prestador que tem mais segurança, diferentemente do que acontece nos serviços públicos. $^{22}$

Com relação a usuários com comorbidades identificou-se, na presente pesquisa, que dos que buscaram a APS, PA e outros serviços, não possuíam, em maioria, outra comorbidade além da tuberculose. Entretanto, os que buscaram o ambulatório de referência e os serviços privados foram aqueles com presença de outras doenças associadas. 
O consumo de tabaco, drogas e álcool foi presente em menos da metade dos relatos da população estudada, o que pode estar relacionado ao estigma sobre o consumo de tais substâncias, e ao fato de admitir frente ao profissional, que utiliza substâncias que causam danos à saúde. Entretanto, estudo realizado em um hospital universitário no Piauí, traz como comorbidades mais relacionadas a tuberculose, o tabagismo e o alcoolismo. ${ }^{23}$

Ainda, cabe considerar que estudos afirmam que fumar além de aumentar o risco da infecção pela tuberculose e a progressão da doença, também pode gerar atrasos na negativação da baciloscopia de escarro. Contudo, ainda a fumaça pode aumentar as chances de os contatos intra-domiciliares contraírem a doença. ${ }^{24}$

\section{Conclusões}

Os resultados deste estudo apontam que o serviço de saúde mais procurado foi o pronto atendimento. Dentre as características das pessoas com TB verificou-se predomínio do sexo masculino, da cor da pele branca, de pessoas que não viviam com companheiro, com escolaridade inferior a 8 anos de estudo, desenvolvendo atividade de emprego formal, com renda entre 1 a 2 salários mínimos. Houve predomínio de comorbidades, prioritariamente, nos grupos que procuraram o primeiro atendimento no ambulatório de referência e nos serviços privados. Quanto ao consumo de álcool, tabaco e drogas ilícitas observou-se que predominou a negativa dos entrevistados, independendo do serviço procurado.

Desse modo, conclui-se que houve diferenças entre o perfil das pessoas com tuberculose de acordo com o comportamento de busca pelos serviços de saúde. Salienta-se que conhecer tais características é essencial para o planejamento de ações para o reconhecimento e captação precoce dos sintomáticos respiratórios no território.

Destaca-se que a baixa procura pela APS evidenciada nos municípios estudados demonstra a insuficiência deste nível de atenção para o atendimento à tuberculose e ressalta a 
importância da mobilização dos gestores municipais no intuito de elaborar estratégias que modifiquem este cenário, e favoreçam o acesso dos sintomáticos de tuberculose à APS.

Acredita-se que diante o fortalecimento da APS e a sua reafirmação como porta de entrada do Sistema Único de Saúde, haverá o controle efetivo acerca de condições sensíveis que ocorrem na comunidade.

O presente estudo teve como limitações o número de questionários que tiveram que ser desconsiderados devido ao primeiro serviço buscado na eminência dos sintomas ter sido deixado em branco, e acredita-se que tal fato se deve ao tempo e ao número de serviços buscados antes de começarem o tratamento para a TB.

Sugere-se que novas pesquisas busquem os motivos que levaram as pessoas com TB a buscarem o PA e o ambulatório de referência em detrimento da APS, para investigar e mapear onde está a quebra no vínculo com o serviço.

\section{Referências}

1. Brasil. Ministério da Saúde. Secretaria de Vigilância em Saúde. Detectar, tratar e curar: desafios e estratégias brasileiras frente à tuberculose. Bol Epidemiol [Internet]. 2015 [acesso em 2018 maio 15];46(9):1-19. Disponível em: http://portalarquivos.saude.gov.br/images/pdf/2015/marco/25/Boletimtuberculose-2015.pdf

2. Brasil. Ministério da Saúde. Secretaria de Vigilância em Saúde. Perspectivas brasileiras para o fim da tuberculose como problema de saúde pública. Bol Epidemiol [Internet]. 2016 [acesso em 2018 maio 18];47(13):1-15. Disponível em: http://portalarquivos2.saude.gov.br/images/pdf/2016/marco/24/2016-009Tuberculose-001.pdf

3. Brasil. Ministério da Saúde. Secretaria de Vigilância em Saúde. Indicadores prioritários para o monitoramento do Plano Nacional pelo Fim da Tuberculose como problema de saúde pública no Brasil. Bol Epidemiol [Internet]. 2017 [acesso em 2018 maio 16];48(8):1-11. Disponível em: http://portalarquivos2.saude.gov.br/images/pdf/2017/marco/23/2017-V-48-N-8-Indicadores-priorit--riospara-o-monitoramento-do-Plano-Nacional-pelo-Fim-da-Tuberculose-como-Problema-de-Sa--de-P-blica-no-Brasil.pdf

4. Brasil. Ministério da Saúde. Secretaria de Vigilância em Saúde. Departamento de Vigilância Epidemiológica. Manual de recomendações para o controle da tuberculose no Brasil [Internet]. Brasília 
Serviço de saúde procurado pelas pessoas com sintomas da tuberculose | 14

(DF): Ministério da Saúde; 2011. [Acesso em 2018 maio 15]. Disponível em: http://bvsms.saude.gov.br/bvs/publicacoes/manual_recomendacoes_controle_tuberculose_brasil.pdf

5. Engel RH, Weiller TH, Farão EMD, Barros IFO, Bordignon J, Luz EMF. Acessibilidade geográfica e organizacional: obstáculos entre usuários com tuberculose e os serviços de saúde. Rev Enferm UFSM [Internet]. 2013 [acesso em 2018 fev 08];3(1):307-14. Disponível em: https://periodicos.ufsm.br/reufsm/article/view/8494

6. Mizuhira VF, Gazetta CE, Vendramini SHF, Ponce MAZ, Wysocki AD, Villa TCS. Procura da atenção básica para o diagnóstico da tuberculose. Arq Ciênc Saúde [Internet]. 2015 [acesso em 2018 fev 07];22(2):94-8. Disponível em: http://www.cienciasdasaude.famerp.br/index.php/racs/article/view/150/74

7. Ponce MAS, Wysocki AD, Scatolin BE, Andrade RLP, Arakawa T, Netto AR, et al. Diagnóstico da tuberculose: desempenho do primeiro serviço de saúde procurado em São José do Rio Preto, São Paulo, Brasil. Cad Saúde Pública [Internet]. 2013 [acesso em 2018 fev 15];29(5):945-54. Disponível em: http://www.scielo.br/pdf/csp/v29n5/12.pdf

8. Dantas DNA, Enders BC, Queiroz AAR, Coura AS, Silva MPM, Menezes RMP. Fatores associados à primeira escolha de local para o diagnóstico da tuberculose. Rev Gaúcha Enferm [Internet]. 2014 [acesso em 2018 jan 09];35(3):75-81. Disponível em: http://www.scielo.br/scielo.php?script=sci_arttext\&pid=S1983$14472014000300075 \& \operatorname{lng}=\mathrm{en}$

9. Centro Estadual de Vigilância em Saúde no Rio Grande do Sul (CEVS-RS). Tuberculose no Rio Grande do Sul. [Internet]. Porto Alegre; 2016 [acesso em 2018 jan 09]. Disponível em: http://www.cevs.rs.gov.br/upload/arquivos/201611/08142336-20161104135226apresentacao-tuberculoseses-rs-04-11-2016.pdf

10. Brasil. Ministério da Saúde. Conselho Nacional de Saúde. Resolução n. 466, de 12 de dezembro de 2012. Aprova as diretrizes e normas regulamentadoras de pesquisas envolvendo seres humanos. Diário Oficial da União. Brasília (DF); 2013 jun 13; Seção 1, p. 59-62.

11. Sasaki NSGMS, Santos MLSG, Vendramini SHF, Ruffino-Netto A, Villa TCS, Chiaravalloti-Neto F. Atrasos na suspeita e no diagnóstico de tuberculose e fatores relacionados. Rev Bras Epidemiol [Internet]. 2015 [acesso em 2018 jan 21];18(4):809-23. Disponível em: http://www.scielo.br/pdf/rbepid/v18n4/1980-5497-rbepid-18-04-00809.pdf

12. Cavalcante EFO, Silva DMGV. Perfil de pessoas acometidas por tuberculose. Rev Rene [Internet]. 2013 [acesso em 2018 jul 04];14(4):720-9. Disponível em: http://www.redalyc.org/pdf/3240/324028459008.pdf

13. Almeida CPB, Skupien EC, Silva DR. Health care seeking behavior and patient delay in tuberculosis diagnosis. Cad Saúde Pública [Internet]. 2015 [acesso em 2018 jan 08];31(2):321-30. Disponível em: http://www.scielo.br/scielo.php?script=sci_arttext\&pid=S0102-311X2015000200321\&lng=en 
14. Paula R, Lefevre F, Lefevre AMC, Galesi VMN, Schoeps D. Por que os pacientes de tuberculose procuram as unidades de urgência e emergência para serem diagnosticados: um estudo de representação social. Rev Bras Epidemiol [Internet]. 2014 [acesso em 2018 fev 20]; 17(3):600-14. Disponível em: http://www.scielo.br/pdf/rbepid/v17n3/pt_1415-790X-rbepid-17-03-00600.pdf

15. Silva WA, Soares YJA, Sampaio JPS, Chaves TVS. Perfil epidemiológico dos casos notificados de tuberculose no Piauí nos anos de 2010 a 2014. Rev Interdisciplinar [Internet]. 2017 [acesso em 2018 jul 04];50(1):110-7. Disponível em: https://revistainterdisciplinar.uninovafapi.edu.br/index.php/revinter/article/view/1095

16. Instituto Brasileiro de Geografia e Estatística. Tabela 3175 - População residente, por cor ou raça, segundo a situação do domicílio, o sexo e a idade [Internet]. 2018 [acesso em 2018 mar 24]. Disponível em: https://sidra.ibge.gov.br/Tabela/3175

17. Gebreweld FH, Kifle MM, Gebremicheal FE, Simel LL, Gezae MM, Ghebreyesus SS, et al. Factors influencing adherence to tuberculosis treatment in Asmara, Eritrea: a qualitative study. J Health Populat Nutr [Internet]. 2018 [acesso em 2018 maio 28];37(1):1-9. Disponível em: https://jhpn.biomedcentral.com/track/pdf/10.1186/s41043-017-0132-y?site=jhpn.biomedcentral.com

18. Araújo SRL, Pereira ISD, Brito NOS, Fonseca, PCB. Perfil epidemiológico da tuberculose pulmonar na cidade do Natal-RN. J Infect Control [Internet]. 2015 [acesso 2018 jul 04];4(1):16-9. Disponível em: http://jic-abih.com.br/index.php/jic/article/view/77

19. Furlan MCR, Marcon SS. Avaliação do acesso ao tratamento de tuberculose sob a perspectiva de usuários. Cad Saúde Colet [Internet]. 2017 [acesso 2018 abr 20];25(3):339-47. Disponível em: http://www.scielo.br/pdf/cadsc/v25n3/1414-462X-cadsc-1414-462X201700030139.pdf

20. Belchior AS, Arcêncio RA, Mainbourg EMT. Diferenças no perfil clínico epidemiológico entre casos novos e casos em retratamento pós abandono. Rev Esc Enferm USP [Internet]. 2016 [acesso em $2018 \mathrm{fev}$ 15];50(4):619-5. Disponível em: http://www.scielo.br/pdf/reeusp/v50n4/pt_0080-6234-reeusp-50-04-0622.pdf

21. Reis SP, Harter J, Lima LM, Vieira DA, Palha PF, Cardozo-Gonzales RI. Aspectos geográficos e organizacionais dos serviços de atenção primária à saúde na detecção de casos de tuberculose em Pelotas, Rio Grande do Sul, 2012. Epidemiol Serv Saúde [Internet]. 2017 [acesso em 2018 fev 15];26(11):1418. Disponível em: http://www.scielo.br/pdf/ress/v26n1/2237-9622-ress-26-01-00141.pdf

22. Tomberg JO, Harter J, Lima LM, Vieira DA, Russo-Gonçalves E, Cardozo-Gonzales RI. Setores público e privado: características sociodemográficas das pessoas com tuberculose e ações de diagnóstico. Rev Enferm UERJ [Internet]. 2017 [acesso em 2018 maio 23]; 25:e22155. Disponível em: http://www.epublicacoes.uerj.br/index.php/enfermagemuerj/article/view/22155 
23. Deus Filho A,Carvalho IMS. Perfil epidemiológico dos pacientes com tuberculose em Hospital Universitário de Teresina-PI. J Ciênc Saúde Hosp Univ Univ Fed Piauí [Internet]. 2018 [acesso em 2018 jul 04];1(1):51-60. Disponível em: http://www.ojs.ufpi.br/index.php/rehu/article/view/6758/pdf

24. Novotny T, Hendrickson E, Soares ECC, Sereno AB, Kiene SM. HIV/AIDS, tuberculose e tabagismo no Brasil: uma sindemia que exige intervenções integradas. Cad Saúde Pública [Internet]. 2017 [acesso em 2018 fev 15];33 Supl 3:e00124215. Disponível em: http://www.scielo.br/pdf/csp/v33s3/1678-4464-csp-33s3-e00124215.pdf

\section{Autor correspondente}

Martina Dias da Rosa Martins

E-mail: martinadrm@hotmail.com

Endereço: rua Leonardo Colares, 389

CEP: $96020-190$

\section{Contribuições de Autoria}

1 - Martina Dias da Rosa Martins

Contribuições: Contribuição substancial para a concepção e planejamento do projeto, obtenção de dados ou análise e interpretação dos dados. Contribuição significativa na elaboração do rascunho ou na revisão crítica do conteúdo. Participação da aprovação da versão final do manuscrito.

\section{2 - Natali Basílio Valerão}

Contribuições: Contribuição substancial para a concepção e planejamento do projeto, obtenção de dados ou análise e interpretação dos dados.

\section{3 - Jéssica Oliveira Tomberg}

Contribuições: Contribuição substancial para a concepção e planejamento do projeto, obtenção de dados ou análise e interpretação dos dados. Contribuição significativa na elaboração do rascunho ou na revisão crítica do conteúdo. Participação da aprovação da versão final do manuscrito.

\section{4 - Lílian Moura de Lima Spagnolo}

Contribuições: Contribuição substancial para a concepção e planejamento do projeto, obtenção de dados ou análise e interpretação dos dados. Contribuição significativa na elaboração do rascunho ou na revisão crítica do conteúdo. Participação da aprovação da versão final do manuscrito.

5- Luciana Nunes soares

Contribuições: Participação da aprovação da versão final do manuscrito. 6- Roxana Isabel Cardozo Gonzales 
17 | Martins MDR, Valerão NB, Tomberg JO, Spagnolo LML, Soares LN, Gonzales RIC

Contribuições: Contribuição substancial para a concepção e planejamento do projeto, obtenção de dados ou análise e interpretação dos dados. Participação da aprovação da versão final do manuscrito.

\section{Como citar este artigo}

Martins MDR, Valerão VB, Tomberg JO, Spagnolo LML, Soares LN, Gonzales RIC. Serviço de saúde procurado pelas pessoas com sintomas da tuberculose. UFSM. 2019 [Acesso em: 2019 jun 15];vol ex:p1p16. DOI:https://doi.org/10.5902/2179769233049 